\title{
M2K. II. A TRIPLE-PLANET SYSTEM ORBITING HIP 57274*
}

\author{
Debra A. Fischer ${ }^{1}$, Eric Gaidos ${ }^{2,3,13}$, Andrew W. Howard ${ }^{4}$, Matthew J. Giguere ${ }^{1}$, John A. Johnson ${ }^{5}$, \\ Geoffrey W. Marcy ${ }^{4}$, Jason T. Wright ${ }^{6,7}$, JefF A. Valenti ${ }^{8}$, Nikolai Piskunov ${ }^{9}$, Kelsey I. Clubb ${ }^{3,13}$, \\ Howard IsaACson $^{3,13}$, Kevin Apps ${ }^{10}$, Sebastien Lepine ${ }^{11}$, Andrew ManN ${ }^{12}$, John Moriarty ${ }^{1}$, John Brewer ${ }^{1}$, \\ Julien F. P. SPRONCK ${ }^{1}$, Christian SCHWAB ${ }^{1}$, AND ANDREW SZYMKOWIAK ${ }^{1}$ \\ ${ }^{1}$ Department of Astronomy, Yale University, New Haven, CT 06511, USA; debra.fischer@yale.edu \\ ${ }^{2}$ Department of Geology and Geophysics, University of Hawaii, Honolulu, HI 96822, USA \\ ${ }^{3}$ Pufendorf Institute for Advanced Studies, Lund University, Lund, Sweden \\ ${ }^{4}$ Department of Astronomy, University of California at Berkeley, Berkeley, CA 94720, USA \\ ${ }^{5}$ Department of Astronomy, California Institute of Technology, Pasadena, CA 91125, USA \\ ${ }^{6}$ Center for Exoplanets and Habitable Worlds, 525 Davey Lab, The Pennsylvania State University, University Park, PA 16803, USA \\ ${ }^{7}$ Department of Astronomy and Astrophysics, 525 Davey Lab, The Pennsylvania State University, University Park, PA 16803, USA \\ ${ }^{8}$ Space Telescope Science Institute, 3700 San Martin Drive, Baltimore, MD 21218, USA \\ ${ }^{9}$ Department of Astronomy and Space Physics, Uppsala University, Box 515, 75120 Uppsala, Sweden \\ 10 75B Cheyne Walk, Surrey RH6 7LR, UK \\ ${ }^{11}$ American Museum of Natural History, New York, NY 10023, USA \\ 12 Institute for Astronomy, University of Hawaii, Honolulu, HI 96822, USA \\ Received 2011 September 5; accepted 2011 October 27; published 2011 December 28
}

\begin{abstract}
Doppler observations from Keck Observatory have revealed a triple-planet system orbiting the nearby K4V star, HIP 57274. The inner planet, HIP 57274b, is a super-Earth with $M \sin i=11.6 M_{\oplus}\left(0.036 M_{\mathrm{Jup}}\right)$, an orbital period of $8.135 \pm 0.004$ days, and slightly eccentric orbit $e=0.19 \pm 0.1$. We calculate a transit probability of $6.5 \%$ for the inner planet. The second planet has $M \sin i=0.4 M_{\text {Jup }}$ with an orbital period of $32.0 \pm 0.02$ days in a nearly circular orbit $(e=0.05 \pm 0.03)$. The third planet has $M \sin i=0.53 M_{\text {Jup }}$ with an orbital period of $432 \pm 8$ days (1.18 years) and an eccentricity $e=0.23 \pm 0.03$. This discovery adds to the number of super-Earth mass planets with $M \sin i<12 M_{\oplus}$ that have been detected with Doppler surveys. We find that $56 \% \pm 18 \%$ of super-Earths are members of multi-planet systems. This is certainly a lower limit because of observational detectability limits, yet significantly higher than the fraction of Jupiter mass exoplanets, $20 \% \pm 8 \%$, that are members of Doppler-detected, multi-planet systems.
\end{abstract}

Key words: planetary systems - stars: individual (HIP 57274)

Online-only material: color figures

\section{INTRODUCTION}

Low-mass $\mathrm{K}$ and $\mathrm{M}$ dwarf stars are important targets for exoplanet surveys because of their proximity and prevalence in the Galaxy. Differences in the number and type of exoplanets orbiting these stars relative to more massive stars reflect conditions in the protoplanetary disk that are important for planet formation. Microlensing surveys suggest that both ice and gas giant planets are common at separations beyond the ice line (Gould et al. 2010). However, the fraction of gas giant planets detected inside the ice line by Doppler surveys is relatively low for late $\mathrm{K}$ and early M dwarfs (Endl et al. 2003; Butler et al. 2006). Fischer et al. (2011) find that the population of giant planets has a precipitous decline for stars redward of $B-V=1.1$, a spectral type of about K5V. Cumming et al. (2008) estimate that relative to FGK stars, $\mathrm{M}$ dwarfs are far less likely to harbor gas giant planets with periods shorter than five years. Johnson et al. (2010) find that $3.4_{-0.9}^{+2.2} \%$ of low-mass stars $\left(M<0.6 M_{\odot}\right)$ have planets with $M \sin i>0.3 M_{\mathrm{Jup}}$ and semimajor axes less than $2.5 \mathrm{AU}$.

The remarkable discovery of more than 1200 planet candidates by the Kepler mission (Borucki et al. 2011) provides statistics for smaller planets and suggests that the reduced planet

\footnotetext{
* Based on observations obtained at the Keck Observatory, which is operated by the University of California.

${ }^{13}$ Visiting Professor.
}

occurrence with later spectral type only applies to gas giant planets. After correcting for the poorer detectability of transits around higher mass stars with larger radii, Howard et al. (2011) find that 20\%-30\% of low-mass stars have planet candidates with Neptune-like radii between 2 and $4 R_{\oplus}$ while the fraction of more easily detected Jupiter-radii planets hovers at a few percent. Howard et al. also see evidence of a rising occurrence of small-radius planets among cooler, less massive stars. Further, Schlaufman \& Laughlin (2011) find that while the planet-metallicity correlation among Sun-like stars is strongest for those hosting large-radius planets, the planet-metallicity correlation among low-mass stars is significant even among hosts of small-radius planets. Doppler surveys of nearby, low-mass stars provide a means of testing whether these correlations hold among stars in the solar neighborhood, and if so, inform the target lists of future planet search efforts.

$\mathrm{K}$ - and M-type stars are especially appealing targets for rocky planet searches in Doppler surveys because the lower stellar mass results in a larger reflex velocity for a given mass planet. Furthermore, chromospheric activity in low-mass stars has less impact on the radial velocities (Isaacson \& Fischer 2010; Lovis et al. 2011). The ubiquity of low-mass stars coupled with more easily detected Doppler signals and lower stellar jitter all make $\mathrm{K}$ and early $\mathrm{M}$ dwarfs desirable targets in the search for rocky planets. However, a caveat has emerged: the inner planetary architectures of low-mass stars may be more complex. Latham et al. (2011) analyzed multi-planet systems detected in transit 
Table 1

Stellar Parameters: HIP 57274

\begin{tabular}{ll}
\hline \hline Parameter & \multicolumn{1}{c}{ Value } \\
\hline$V$ & \\
$B-V$ & 8.96 \\
Spec type & 1.11 \\
Distance $(\mathrm{pc})$ & $\mathrm{K} 4 \mathrm{~V}$ \\
$M_{V}$ & 25.92 \\
Bol. corr. & 6.89 \\
$L_{*}\left(L_{\odot}\right)$ & -0.50 \\
$M_{*}\left(M_{\odot}\right)$ & $0.19(0.01)$ \\
$R_{*}\left(R_{\odot}\right)$ & $0.73(0.05)$ \\
Age $(\mathrm{Gyr})$ & $0.68(0.03)$ \\
$T_{\mathrm{eff}}(\mathrm{K})$ & $7.87(5)$ \\
$\log g$ & $4640(100)$ \\
{$[$ Fe $/ \mathrm{H}]$} & $4.71(0.1)$ \\
$v$ sin $i\left(\mathrm{~km} \mathrm{~s}{ }^{-1}\right)$ & $+0.09(0.05)$ \\
$S_{\mathrm{HK}}$ & $0.5(0.5)$ \\
$\log R_{\mathrm{HK}}^{\prime}$ & $0.39(0.02)$ \\
$P_{\text {rot }}$ & -4.89 \\
$\operatorname{Radial~velocity~}\left(\mathrm{km} \mathrm{s}^{-1}\right)$ & 45 days \\
& -4.7
\end{tabular}

with the Kepler mission and found that solar-type and hotter stars are more common hosts of single transiting planets, while multi-planet systems are more often detected around cooler stars. Among the 170 multi-planet systems detected by the Kepler mission, $78 \%$ contain planets no larger than Neptune; close-in gas giant planets are far less common in multi-planet systems (Latham et al. 2011). This has profound implications for Doppler surveys: the challenge of detecting the small velocity amplitudes of Neptune-like planets will be compounded by the need to deconvolve multiple signals of similar amplitude. For both of these reasons, a larger number of observations over a longer interval of time are required to resolve the components of these planetary systems.

To better understand the frequency and architectures of planetary systems around low-mass stars, we began " $\mathrm{M} 2 \mathrm{~K}$ " (Apps et al. 2010), a Doppler survey of $\mathrm{M}$ and $\mathrm{K}$ dwarf stars drawn from the SUPERBLINK proper motion survey (Lepine \& Shara 2005; Lepine \& Gaidos 2011). Here, we report the detection of a triple-planet system orbiting HIP 57274 comprised of a super-Earth mass planet and two planets that are likely gas giants.

\section{OBSERVATIONS AND DATA}

Doppler observations are carried out with the Keck 10 m telescope and the High Resolution Echelle Spectrometer (HIRES) spectrograph (Vogt et al. 1994). An iodine cell is used to provide the wavelength solution and sampling of the line-spread function to model the Doppler shift in the stellar spectra (Butler et al. 1996). The B5 decker on HIRES provides a spectral resolution of about 55,000 and an exposure meter terminates the observations when a target signal-to-noise ratio of about 200 is achieved. Most of the M2K stars are fainter than $V=9$, requiring exposure times of 10-15 minutes. We have acquired three or more Doppler measurements for more than 170 stars, with formal measurement uncertainties of about $1.5 \mathrm{~m} \mathrm{~s}^{-1}$.

\subsection{HIP57274}

HIP 57274 (GJ 439) has an apparent magnitude of $V=8.96$, color $B-V=1.111$, and parallax of $38.58 \pm 1$ mas according to the Hipparcos catalog (ESA 1997; van Leeuwen 2007). This
Table 2

Radial Velocities for HIP 57274

\begin{tabular}{lrcc}
\hline \hline JD-2440000 & $\begin{array}{c}\mathrm{RV} \\
\left(\mathrm{m} \mathrm{s}^{-1}\right)\end{array}$ & $\begin{array}{c}\sigma_{\mathrm{RV}} \\
\left(\mathrm{m} \mathrm{s}^{-1}\right)\end{array}$ & $S_{\mathrm{HK}}$ \\
\hline 14806.13297 & 0.65 & 1.34 & 0.469 \\
14807.14718 & -2.97 & 1.88 & 0.469 \\
14809.15850 & -1.80 & 1.26 & 0.468 \\
15190.15800 & -14.89 & 1.18 & 0.409 \\
15198.16272 & -7.39 & 1.29 & 0.403 \\
15232.04776 & 11.50 & 1.26 & 0.411 \\
\hline
\end{tabular}

yields a distance of $25.9 \mathrm{pc}$ and absolute visual magnitude of $M_{V}=6.89$. We carried out spectral synthesis modeling of the iodine-free template spectrum using Spectroscopy Made Easy (SME; Valenti \& Piskunov 1996; Valenti \& Fischer 2005) to determine stellar parameters. Following the method described in Valenti et al. (2009), the initial parameters derived with SME were used as input for interpolation of the Yonsei-Yale (Y2) isochrones (Demarque et al. 2004), which returns a new value for $\log g$. We then ran an iterative loop, fixing $\log g$ to the isochrone value and running a new SME model fit. The other (free) stellar parameters change in response to the fixed surface gravity, so subsequent isochrone interpolations produce a slightly different value for $\log g$. We continue the iteration until the output $\log g$ from the isochrones does not change by more than 0.001 dex from the previous iteration. This provided the following spectroscopic parameters: $T_{\text {eff }}=4640 \pm 100 \mathrm{~K}, v \sin i=$ $0.5 \pm 0.5 \mathrm{~km} \mathrm{~s}^{-1},[\mathrm{Fe} / \mathrm{H}]=+0.09 \pm 0.05, \log g=4.71 \pm$ 0.1 , and Y2 isochrone models for a stellar mass of $0.73 \pm$ $0.05 M_{\odot}$, a radius of $0.68 \pm 0.03 R_{\odot}$, and an age of $7.87 \pm 5 \mathrm{Gyr}$. The brightness is consistent with any main-sequence age. The spectral classification is listed as K8V in the Hipparcos catalog, although the $B-V$ color, spectroscopic temperature, and derived mass are more consistent with a somewhat earlier spectral type. One of us (S.L.) has obtained a medium-resolution spectrum with the Mark III spectrograph at the MDM $1.3 \mathrm{~m}$ telescope; the absence of a clear TiO absorption band head redward of $7000 \AA$ rules out spectral classifications of $\mathrm{K} 5 \mathrm{~V}$ or later. The hint of a weak $\mathrm{TiO}$ band absorption is consistent with the K4V classification of Gray et al. (2003), and we adopt this spectral classification here. The stellar parameters for HIP 57274 are summarized in Table 1.

\subsection{Chromospheric Activity and Velocity Jitter}

Isaacson \& Fischer (2010) determined the chromospheric activity of 2630 stars observed at Keck by measuring the emission in the cores of the $\mathrm{Ca}$ II $\mathrm{H}$ and $\mathrm{K}$ lines relative to adjacent continuum regions. These $S_{\mathrm{HK}}$ values were calibrated to the long-standing $S_{\mathrm{HK}}$ values from the Mt. Wilson program (Duncan et al. 1991). Using their technique, we measure a mean $S_{\mathrm{HK}}=0.38$ for HIP 57274. The individual measurements of $S_{\mathrm{HK}}$ are listed in the last column of Table 2, along with the radial velocity measurements.

Cooler stars typically have larger $S_{\mathrm{HK}}$ values than solar-type stars because of weaker continuum in the near-UV. Therefore, $S_{\mathrm{HK}}$ values should not be directly compared for stars of different spectral types. Noyes et al. (1984) correct for the photospheric contributions to produce a normalized activity metric, $\log R_{\mathrm{HK}}^{\prime}$. Chromospheric activity is tied to dynamo-driven magnetic fields and decreases as the star ages and spins down. Noyes et al. (1984) made use of this activity-rotation correlation and calibrated $\log R_{\mathrm{HK}}^{\prime}$ to rotational periods for stars in open 


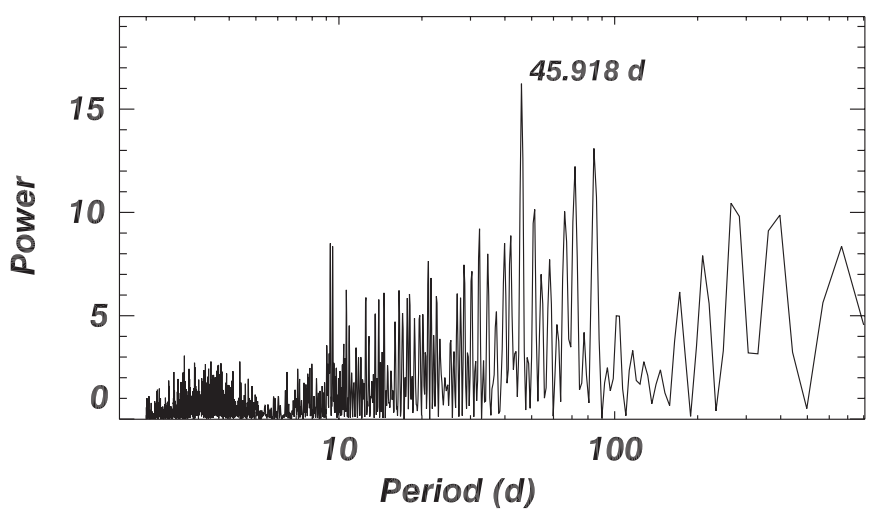

Figure 1. Periodogram of the $S_{\mathrm{HK}}$ chromospheric activity metric shows power at 45.9 days, consistent with the rotation period prediction from the activity calibration of Noyes et al. (1984). Photometric follow-up will help to confirm this result.

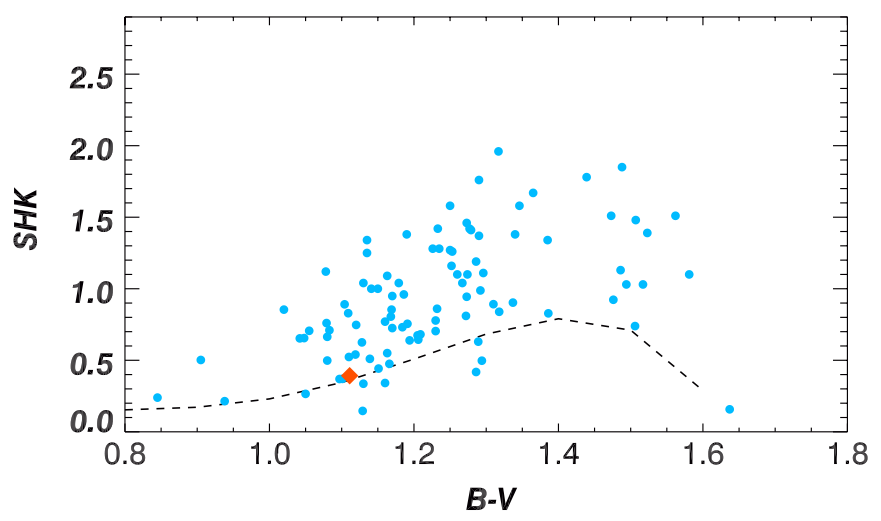

Figure 2. Emission in the core of the $\mathrm{Ca} I \mathrm{II}$ and $\mathrm{K}$ lines is a signature of chromospheric activity that is parameterized as an $S_{\mathrm{HK}}$ value for each star and plotted above as a function of $B-V$. The dashed line represents the baseline for low-activity stars from Isaacson \& Fischer (2010). Inactive stars fall near the dashed line with $\Delta S_{\mathrm{HK}} \sim 0.0$ and active stars have a large $\Delta S_{\mathrm{HK}}$. A filled diamond is used to show the $S_{\mathrm{HK}}$ measurement for HIP 57274.

(A color version of this figure is available in the online journal.)

clusters of different ages. Using the Noyes relation, we derive $\log R_{\mathrm{HK}}^{\prime}=-4.89$, indicating low activity for HIP 57274 and $P_{\text {rot }} \sim 45$ days, implying a relatively old age in the broad range allowed by the observed brightness. This is also in agreement with the periodogram of the $S_{\mathrm{HK}}$ activity indicator (Figure 1), which has power at 45.9 days. We caution that both $\log R_{\mathrm{HK}}^{\prime}$ and $P_{\text {rot }}$ were calibrated by Noyes et al. (1984) using stars with $0.4<B-V<1.0$ and rotational periods shorter than 30 days; HIP 57274 falls outside both of these properly calibrated ranges and therefore our derived $\log R_{\mathrm{HK}}^{\prime}$ and rotational period should be considered to be rough estimates. The rotation period will need to be verified with photometric observations during the next observing season for this star.

Because the activity calibration for stars redward of $B-V=$ 1.0 is an extrapolation, Isaacson \& Fischer (2010) established a differential activity measurement, $\Delta S_{\mathrm{HK}}$, and evaluated the impact of chromospheric activity on radial velocities in four separate ranges of $B-V$. Following their method, we plot the $S_{\mathrm{HK}}$ index for the 170 stars observed on the M2K program with $B-V$ color from 0.8 to 1.6 (Figure 2). The dashed line in this plot is taken from Isaacson \& Fischer (2010) and indicates the baseline $S_{\mathrm{HK}}$ values for low-activity stars. $\Delta S_{\mathrm{HK}}$ is the difference between this baseline activity level and the mean $S_{\mathrm{HK}}$ for a given star. Active stars float high above the baseline values while

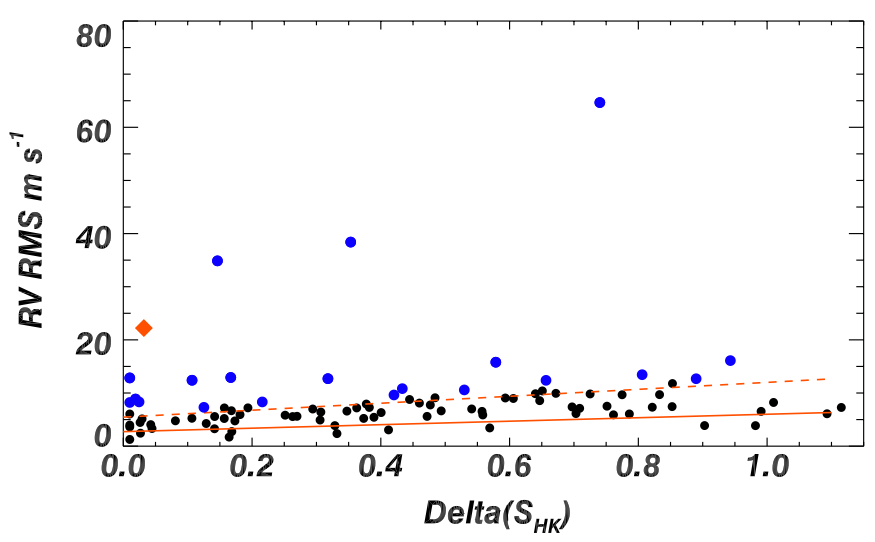

Figure 3. rms velocity scatter is plotted as a function of activity. No trends in the velocity data were removed. The solid red line is a linear fit to the bottom 20 th percentile velocity scatter. Stars with more than $3 \sigma$ jitter (the dashed line) are indicated with blue dots and represent prospective planet candidates. HIP 57274 is represented by the filled diamond.

(A color version of this figure is available in the online journal.)

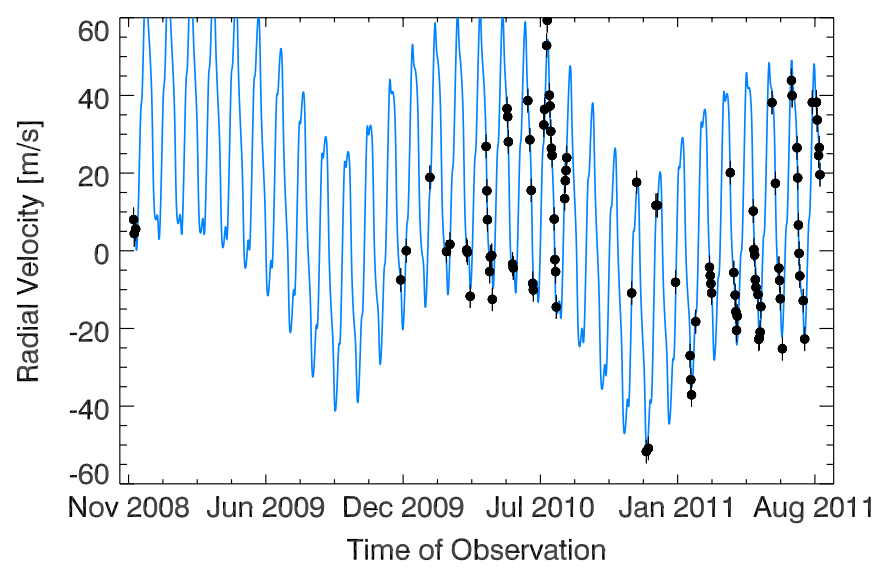

Figure 4. Time series data for HIP 57274 are shown with a Keplerian model for three planetary signals plus a linear trend. The model fit has an rms in the residual velocities of $3.15 \mathrm{~m} \mathrm{~s}^{-1}$ and $\chi_{v}^{2}=1.06$, with an assumed jitter of $2.8 \mathrm{~m} \mathrm{~s}^{-1}$ added in quadrature to the internal errors.

(A color version of this figure is available in the online journal.)

chromospherically quiet stars are closer to the dashed line in Figure 2.

In Figure 3, we plot velocity rms as a function of excess chromospheric activity, $\Delta S_{\mathrm{HK}}$, for all of the observed M2K stars. We fit a linear function to the lower 20th percentile velocity scatter and interpret this (red solid line overplotted in Figure 3) as the quadrature sum of internal errors and jitter (where jitter is both instrumental and astrophysical). The rms scatter for inactive stars with $\Delta S_{\mathrm{HK}} \sim 0.0$ is $2.38 \mathrm{~m} \mathrm{~s}^{-1}$, and given the typical internal errors of $1.5 \mathrm{~m} \mathrm{~s}^{-1}$, this implies a minimum jitter of $1.45 \mathrm{~m} \mathrm{~s}^{-1}$. We measure $\Delta S_{\mathrm{HK}}=0.03$ for HIP 57274 , suggesting a low stellar jitter of $\sim 1.5 \mathrm{~m} \mathrm{~s}^{-1}$.

\subsection{Doppler Observations and Keplerian Model}

We have obtained 99 observations of HIP 57274 with a mean signal-to-noise ratio of 200 and an average exposure time of $360 \mathrm{~s}$. The mean formal measurement errors are $1.23 \mathrm{~m} \mathrm{~s}^{-1}$ (Table 2). Figure 4 shows the time series data, overplotted with our Keplerian model for a triple-planet system. We used the partially linearized Levenberg-Marquardt algorithm (Wright \& Howard 2009) built into the Keplerian Fitting Made Easy (KFME) program (Giguere et al. 2011) to model the data. The 


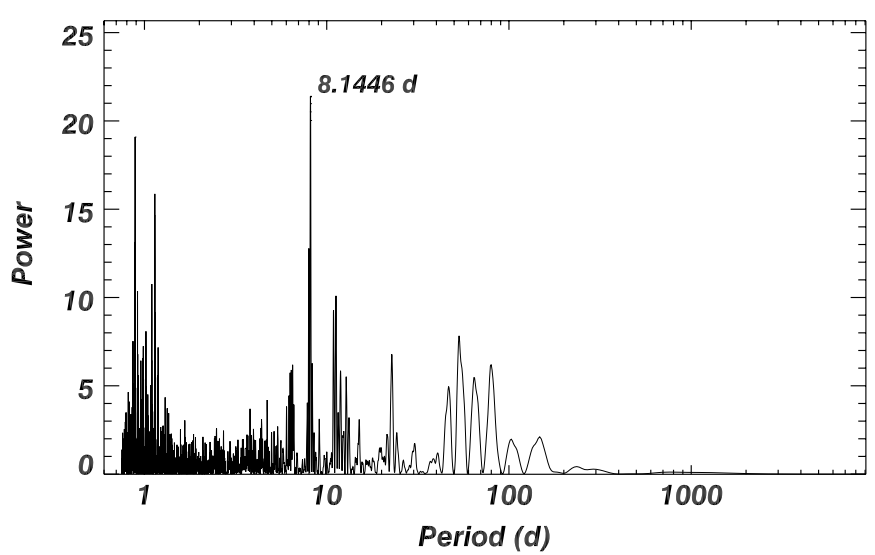

Figure 5. After subtracting the linear trend and the best-fit theoretical velocities for the two outer planets, the periodogram of the residuals shows significant power at 8.135 days. The signal of this inner planet, which we designate as HIP 57274 b, has a FAP $<0.0001$. The two peaks just below and above a period of 1.0 day are aliases of the 8.14 day peak.

best-fit Keplerian model for three planets includes a trend of $-0.026 \mathrm{~m} \mathrm{~s}^{-1} \mathrm{day}^{-1}$. Parameter uncertainties were calculated with a bootstrap Monte Carlo analysis (Marcy et al. 2005) in KFME (Giguere et al. 2011), which iteratively fits the data with a best-fit model, then adds the scrambled residuals back to the theoretical velocities before refitting.

The planet with the shortest period completes one orbit in $8.135 \pm 0.004$ days and induces a velocity amplitude of $4.64 \pm 0.46 \mathrm{~m} \mathrm{~s}^{-1}$. With a stellar mass of $0.73 M_{\odot}$, we derive a planet mass $M \sin i=11.6 M_{\oplus}$ and a semimajor axis of $0.07 \mathrm{AU}$. The orbital eccentricity is $0.187 \pm 0.10$ and the argument of periastron passage $\omega \sim 82^{\circ}$. Because the velocity amplitude is small compared to the uncertainties and stellar jitter, the eccentricity for this planet is poorly constrained, however the signal itself is unambiguous. Figure 5 shows the periodogram of the residual velocities of HIP 57274 after removing the other two planets and linear trend described below. We carried out a Monte Carlo test to determine the false alarm probability (FAP) of the periodogram power. In this test, 10,000 trials were carried out where the best-fit (triple Keplerian and trend) model was subtracted and the residual velocities were scrambled before being added back to the theoretical velocities and refitting. In these 10,000 trials, a peak at least as high as observed was never found in the residuals to the fit of the two more massive planets, yielding a FAP less than $10^{-4}$. Figure 6 shows the phase-folded velocities of HIP 57274 b overplotted with the theoretical Keplerian model after removing the signals from the two additional planets and the linear trend.

We calculated the prospective time of transit, transit duration, and transit probability using KFME (Giguere et al. 2011). The transit ephemeris is $2455801.779 \pm 0.27$ HJD or 2011 August 28 06:41:40.7 UT. The next transit observable from Mauna Kea occurs at 3 AM Hubble Space Telescope (HST) on 2012 January 13 , except that the uncertainty in the transit time is more than $6 \mathrm{hr}$. The duration of the prospective transit would be $3.08 \pm 0.356 \mathrm{hr}$ and the geometric probability that this planet will transit is $6.5 \%$.

The middle planet in this system has an orbital period of $32.0 \pm 0.02$ days. The best-fit model for this planet has a nearly circular orbit, with an eccentricity of $0.05 \pm 0.02$. The velocity amplitude is $32.4 \pm 0.6 \mathrm{~m} \mathrm{~s}^{-1}$, implying a planet with $M \sin i=$ $130 M_{\oplus}$ or $0.41 M_{\text {Jup }}$ and an orbital radius of $0.18 \mathrm{AU}$. The phase-folded data and model for HIP $57274 \mathrm{c}$ is shown in

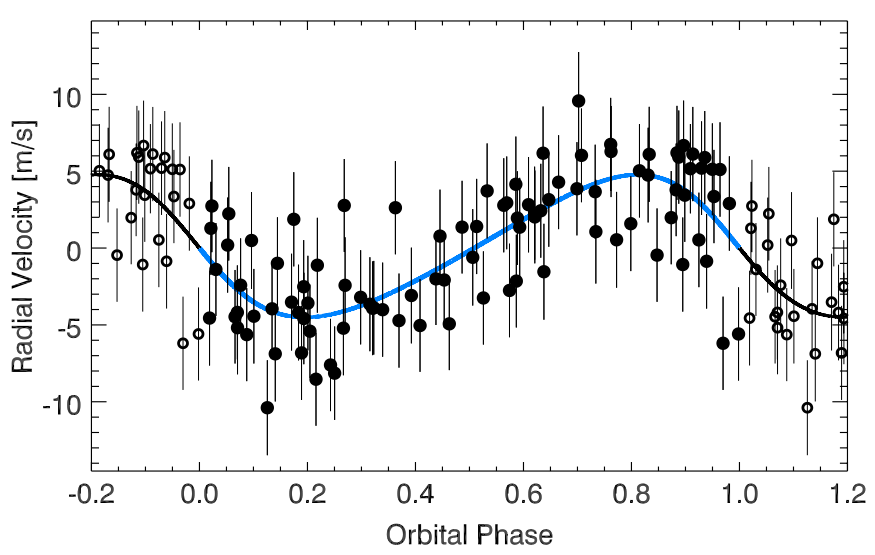

Figure 6. Phase-folded radial velocities for HIP 57274b are shown after removing Keplerian signals from the outer two planets and subtracting a linear trend. The Keplerian model is plotted with a solid line and has an orbital period of $8.135 \pm 0.005$ days, orbital eccentricity $e=0.19 \pm 0.1$, and $M \sin i=$ 11.6 $M_{\oplus}$.

(A color version of this figure is available in the online journal.)

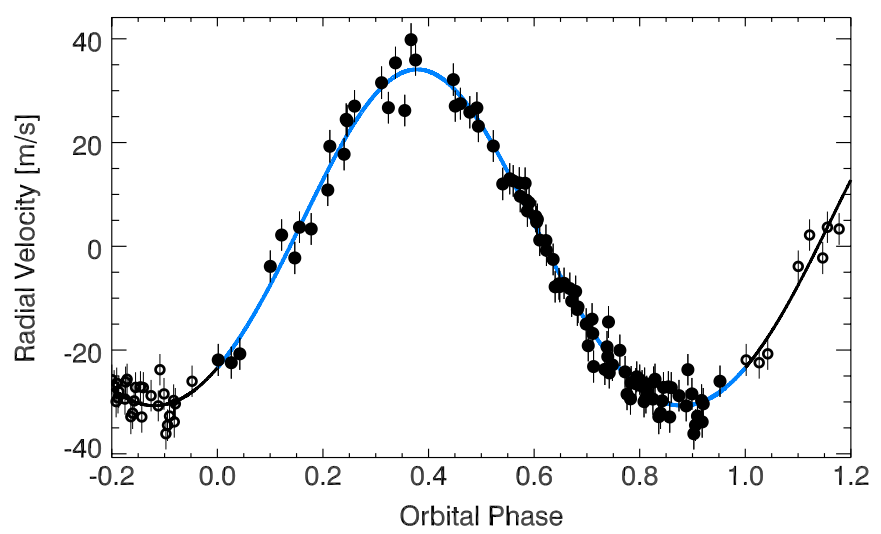

Figure 7. Phase-folded radial velocities for HIP 57274c are shown with theoretical Keplerian velocities for the inner and outer planets and the linear trend subtracted. The Keplerian model has a period of $32.0 \pm 0.05$ days, an eccentricity of $0.05 \pm 0.03$, and $M \sin i=130 M_{\oplus}$ or $0.41 M_{\text {Jup }}$.

(A color version of this figure is available in the online journal.)

Figure 7 after subtracting theoretical velocities for the linear trend and the inner and outer planets. The prospective ephemeris time is $2454793.035 \pm 0.176 \mathrm{HJD}$, although the longer orbital period for the middle planet results in a lower $2.7 \%$ transit probability.

The third planet, HIP $57274 d$, has an orbital period of $432 \pm 8$ days, a velocity amplitude of $18.2 \pm 0.5 \mathrm{~m} \mathrm{~s}^{-1}$, and an orbital eccentricity of $0.27 \pm 0.05$. The planet mass is $M \sin i=$ $0.53 M_{\text {Jup }}$, and the semimajor axis of the orbit is a familiar 1.01 AU. Figure 8 shows the time series velocities and Keplerian model for this planet after removing the inner two planets and the linear trend. The velocity rms for the triple-planet fit is $3.15 \mathrm{~m} \mathrm{~s}^{-1}$. However, if we adopt the predicted jitter of $1.5 \mathrm{~m} \mathrm{~s}^{-1}$, we find that $\chi_{v}^{2}=2.7$, indicating that the model does not fully describe our observations. The Keplerian models for planets b, $\mathrm{c}$, and $\mathrm{d}$ are summarized in Table 3 .

To check for additional planets in the system, we subtracted the theoretical velocities for the linear trend and three Keplerian models. Figure 9 shows a periodogram of the residual velocities, with significant peaks at 1.019 days and 52.996 days that could be aliases of each other, due to the diurnal cadence: $1.0+$ $1 . / 52.996=1.019$ days. This predicts the presence of a second peak at $1.0-1 . / 52.996-0.981$, and we see a second peak 
Table 3

Orbital Parameters for HIP 57274

\begin{tabular}{|c|c|c|c|}
\hline Parameter & $\mathrm{b}$ & $\mathrm{c}$ & $\mathrm{d}$ \\
\hline$P$ (days) & $8.1352(0.004)$ & $32.03(0.02)$ & $431.7(8.5)$ \\
\hline$T_{\mathrm{p}}-2.44 \times 10^{6}(\mathrm{JD})$ & $14801.015(1.3)$ & $15785.208(9.5)$ & $15108.116(14)$ \\
\hline ecc & $0.187(0.10)$ & $0.05(0.02)$ & $0.27(0.05)$ \\
\hline$\omega(\operatorname{deg})$ & $81(59)$ & $356.2(120.0)$ & $187.2(5)$ \\
\hline$K_{1}\left(\mathrm{~m} \mathrm{~s}^{-1}\right)$ & $4.64(0.47)$ & $32.4(0.6)$ & $18.2(0.5)$ \\
\hline$M \sin i\left(M_{\oplus}\right)$ & $11.6(1.3)$ & $130(3)$ & $167.4(8)$ \\
\hline$a_{\text {rel }}(\mathrm{AU})$ & 0.07 & 0.178 & 1.01 \\
\hline$T_{c}(\mathrm{HJD}-2.44 \mathrm{e} 6)$ & $15801.779(0.271)$ & $15793.035(0.176)$ & \\
\hline$T_{\text {duration }}(\mathrm{hr})$ & $3.08(0.35)$ & $5.88(0.1)$ & \\
\hline$t_{\text {prob }}$ & $6.5 \%$ & $2.7 \%$ & \\
\hline Trend $\left(\mathrm{m} \mathrm{s}^{-1}\right.$ day $\left.^{-1}\right)$ & $-0.026(0.002)$ & & \\
\hline $\operatorname{Avg~S/N~}$ & 225 & & \\
\hline $\operatorname{rms}\left(\mathrm{m} \mathrm{s}^{-1}\right)$ & 3.15 & & \\
\hline Nobs & 99 & & \\
\hline$\chi_{v}^{2}$ & 1.06 & & \\
\hline $\operatorname{Jitter}\left(\mathrm{m} \mathrm{s}^{-1}\right)$ & 2.8 & & \\
\hline
\end{tabular}

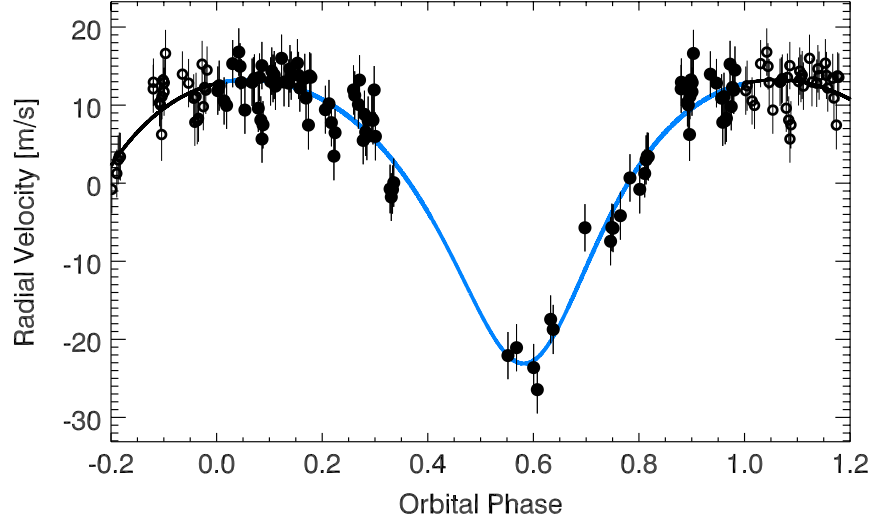

Figure 8. Phase-folded data for HIP 57274d is shown with the two inner planets and a linear trend removed. The best-fit Keplerian model has a period of $431.7 \pm 8.5$ days, $M \sin i=0.53 M_{\mathrm{Jup}}$, and an eccentricity of $0.27 \pm 0.05$. (A color version of this figure is available in the online journal.)

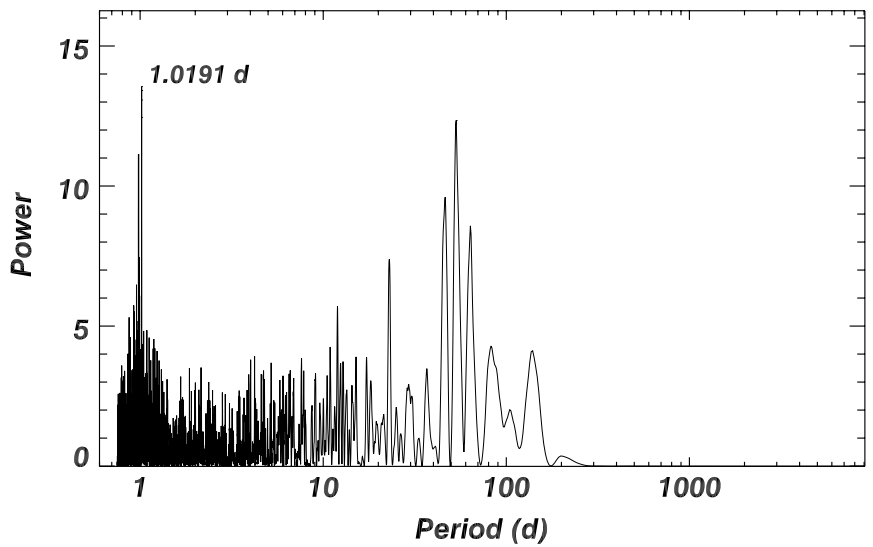

Figure 9. After subtracting the linear trend and the best-fit theoretical velocities for three planets, the periodogram of the residuals shows significant power near 1.0193 and 53 days. These peaks are likely aliases of each other from the diurnal cadence, and the peak near 53 days could be associated with spots on the surface of this cool star.

in the periodogram at 0.981 days. Interestingly, the $\sim 53$ day peak has been increasing in strength. Unlike the periodograms for the three planets that we modeled (HIP 57472b, c, and d), the 53 day peak is flanked by two additional peaks. We suspect

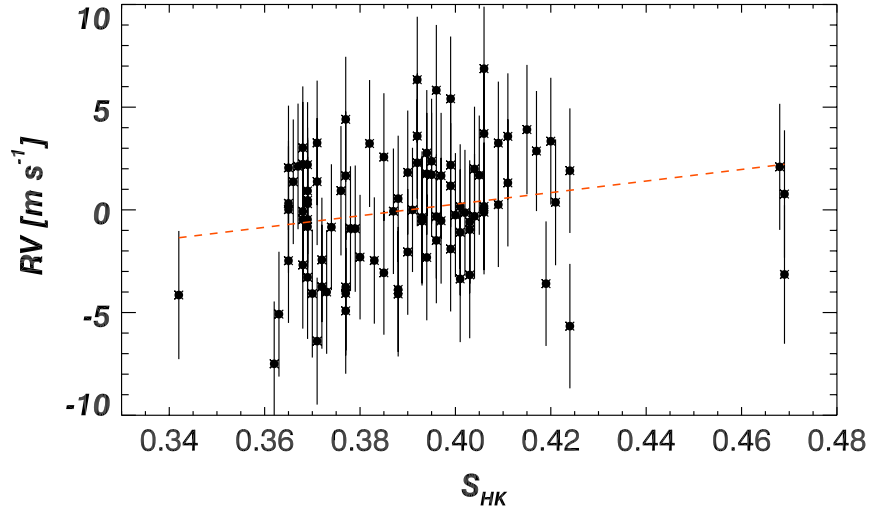

Figure 10. Residual velocities to the triple-planet model are plotted as a function of $S_{\mathrm{HK}}$ activity measurements and fit with a first-order polynomial and do not show a significant trend.

(A color version of this figure is available in the online journal.)

that this signal may be caused by spots rotating on the surface of the star which reduce flux on the approaching blueshifted edge of the star and then on the receding redshifted edge of the star. Physically, this would produce a line profile asymmetry that could be spuriously modeled as reflex motion due to a planet. We checked to see if the residual velocities to the full triple-planet fit were correlated with the activity measurements but found only an insignificant trend (Figure 10, dashed line). We tried detrending the velocities with the linear fit shown in Figure 10, however this only slightly reduced the periodogram power in the residual velocities. If we blindly fit this periodic signal with a Keplerian model, we derive a period of 53.2 days with an amplitude of $2.6 \mathrm{~m} \mathrm{~s}^{-1}$, and the residuals drop to $2.63 \mathrm{~m} \mathrm{~s}^{-1}$ with a $\chi_{v}^{2}=1.28$. Interpreting this signal as an additional noise source from coherent spots, we add the $2.63 \mathrm{~m} \mathrm{~s}^{-1}$ signal in quadrature with the expected jitter of $1.5 \mathrm{~m} \mathrm{~s}^{-1}$ to obtain a revised jitter estimate of $2.8 \mathrm{~m} \mathrm{~s}^{-1}$. This changes $\chi_{v}^{2}$ to 1.06 for the model of a triple-planet system plus a linear trend. If the 53 day signal originates from star spots, then it should have a detectable photometric signal. We have started a photometric campaign to check for spot modulation and to search for the transit signal of the inner 8.135 day planet. In addition to the photometric observations, we will continue to obtain Doppler 
measurements to better understand the origin of the 53 day signal.

\subsection{Dynamical Stability}

To assess the stability of the HIP 57274 system, we ran an ensemble of dynamical simulations of the triple-planet system with the hybrid symplectic integrator code Mercury 6 (Chambers 1999). Orbital parameters for each body were calculated from the values in Table 1 assuming Gaussiandistributed errors (with a truncation at 0.0 for eccentricity). We ran 20 simulations with $\sin i=1$ (minimum mass case) and 15 simulations with $\sin i=0.3$ (95\% of random orientations will have a $\sin i$ greater than this value) and a mass of $0.73 M_{\odot}$ for the central star. For radii and thus collision probabilities, we assumed mean densities of $6.0,1.0$, and $1.0 \mathrm{~g} \mathrm{~cm}^{-3}$ for the $\mathrm{b}$, $\mathrm{c}$, and d planets, which would be typical for the densities of rocky and gas giant planets. The time step was set to 0.365 days (4.5\% of the orbital period of HIP 57274b) and each simulation was run for $10 \mathrm{Myr}$. In none of the 35 independent simulations did collisions or ejections occur. We conclude that the system is stable regardless of its inclination.

The orbital periods of planets B and C are within $1.6 \%$ of a 1:4 mean-motion commensurability and thus we examined the possibility that they might be in resonance. The resonance conditions are that the libration angle

$$
\phi=p L_{B}-q L_{C}-m \omega_{B}-n \Omega_{B}-r \omega_{C}-s \Omega_{C},
$$

where $L$ is the mean anomaly, $\omega$ is the longitude of periastron, $\Omega$ is the longitude of the ascending node, and $p, q, m, n, r, s$ are small integers, osculates around a fixed value rather than circulates over $0-2 \pi$. In this case, $p=1$ and $q=4$. We performed 10 simulations as previously described, varying the initial orbital elements according to the solution uncertainties and recording the osculating elements every $10^{3}$ years. In every simulation $\phi$ orbits with a period of $\sim 1.5$ years. We also examined apsidal resonance between $\mathrm{B}$ and $\mathrm{C}$, but found that $\omega_{B}-\omega_{C}$ also circulates, with a period of $\sim 650$ years. Thus there is no evidence that $\mathrm{B}$ and $\mathrm{C}$ are in an orbital resonance, but the question should be revisited when additional radial velocity data permit more precise orbit determinations.

To further evaluate a planetary explanation for the signal at 53 days, we performed 20 additional dynamical simulations spanning $10 \mathrm{Myr}$ with a four-planet solution, including an "e" planet with an orbital period of 53.3 days and a minimum mass of 12.3 Earths. Other details of the simulations were the same as before. In ten simulations, $\sin i=1$, and in the other ten, $\sin i=0.31$. In eight of the first set, e collided with either $\mathrm{c}$ or $\mathrm{d}$ (four instances each) within $10 \mathrm{Myr}$. In all ten runs of the second set, e collided with either c (six instances) or d (six instances) in under $10^{5}$ years. These results strongly disfavor the existence of a fourth planet on a 53 day orbit, at least with a mass sufficient to explain the Doppler signal at that period.

\section{SUMMARY AND DISCUSSION}

Here we present a triple-planet system orbiting the late $\mathrm{K}$ dwarf star, HIP 57274. The inner planet orbits in 8.135 days and has a mass $M \sin i=11.6 M_{\oplus}$. The orbit is slightly eccentric with periastron directed toward our line of site. We calculate a transit probability of $6.5 \%$ with a putative ephemeris time, $T_{c}=$ $2455801.776 \pm 0.338$, and a duration of $3.08 \pm 0.35 \mathrm{hr}$. The second planet orbits in 32 days and has $M \sin i=130.0 M_{\oplus}$. The third planet has an orbital period of 432 days with a semimajor

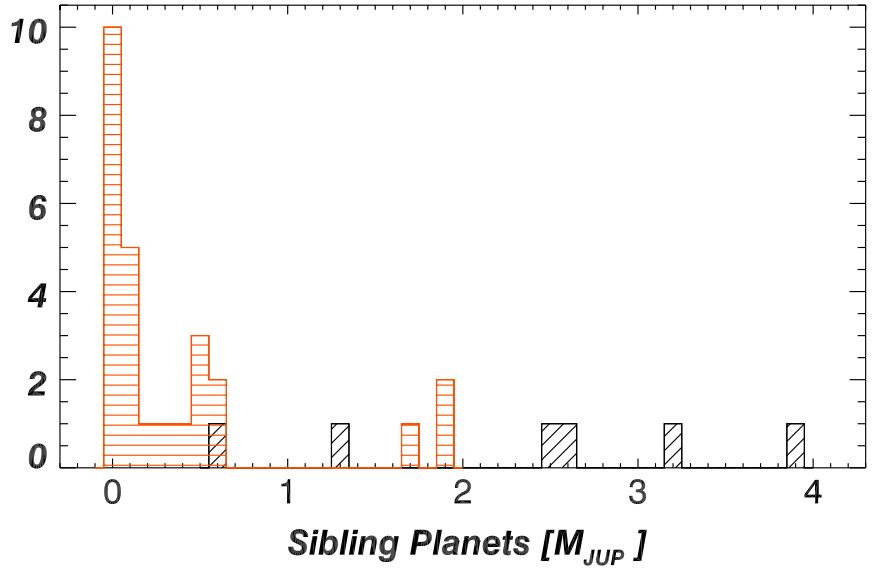

Figure 11. Excluding the "primary" planets in our two subsamples, we show the distribution of sibling planets in the multi-planet systems with super-Earths (red, horizontal hashed histogram) and Jovian planets (black, diagonal hashed histogram).

(A color version of this figure is available in the online journal.)

axis of $1.01 \mathrm{AU}$ and $M \sin i=167.4 M_{\oplus}$. The nominal habitable zone of this $\mathrm{K}$ star, corresponding to $0.95-1.3 \mathrm{AU}$ around the Sun (Kasting et al. 1993) and adjusting for the lower luminosity, lies between 0.41 and $0.57 \mathrm{AU}$ or orbital periods of 110 and 180 days. No significant periodic signals lie within this range (Figure 9).

With the addition of HIP 57274b, there are now 25 planets with $M \sin i<12.0 M_{\oplus}$ listed in the Exoplanet Orbit Database or EOD (Wright et al. 2011). It is probable that these 25 planets are super-Earths or Neptunes rather than gas giant planets. Importantly, only 8 of the 25 (currently) appear to reside in single-planet systems. The remaining 17 low-mass planets are constituents of 10 multi-planet systems. Counting systems instead of planets and applying Poisson error bars (i.e., the percentage of single- or multiple-planet systems divided by the square root of the number of planets), we find that $44 \% \pm 16 \%$ of these hosts of low-mass planets have only one known planet (at the current level of Doppler detectability), while $55.6 \% \pm 17.6 \%$ have multiple planets. To restate, more than half of the Dopplerdetected super-Earths are detected as members of multi-planet systems.

To compare the architectures of planetary systems containing super-Earths with those containing gas giant planets, we extracted all 36 planets from Doppler surveys with $M \sin i$ between 1.0 and $1.5 M_{\text {Jup }}$ orbiting main-sequence stars in the EOD. In this Jovian-mass subsample, there were 30 single-planet systems $(86 \% \pm 16 \%)$ and 5 multi-planet systems $(14 \% \pm 6 \%)$. This result is not particularly sensitive to the arbitrary range of exoplanet mass: in a subsample of 63 planets with $M \sin i$ from 1.5 to $2.5 M_{\text {Jup }}, 73 \% \pm 11 \%$ systems were single. Another sample cut of 41 planets with $M \sin i$ from 3.0 to $6.0 M_{\text {Jup }}$ yielded $83 \% \pm 14 \%$ single-planet systems. Taking an average of these three subsamples, roughly $80 \% \pm 10 \%$ of the Doppler-detected Jupiters hosts have only one known planet and about $20 \% \pm 8 \%$ of Jupiter hosts have multiple planets. This can be compared with the estimate of Wright et al. (2009) who find that at least $28 \%$ of planets are in multiple systems. Since they count planets of all masses detected before 2009, that result is not inconsistent with our estimate. Relative to super-Earths, Doppler surveys detect significantly fewer multi-planet systems with gas giant planets.

In Figure 11, we consider the sibling planets in these multiplanet architectures and compare the super-Earth and Jupiter 


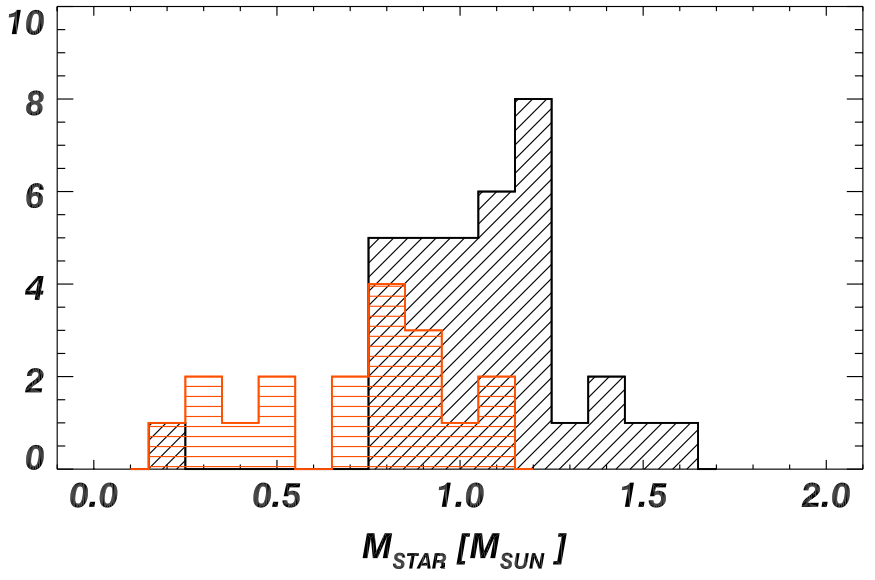

Figure 12. Stellar hosts of detected super-Earth planets (red, horizontal histogram) are systematically lower in mass than the stellar hosts of Jupiter mass planets (black diagonal lines).

(A color version of this figure is available in the online journal.)

subsamples. A total of 26 planets accompany the 10 super-Earths in known multi-planet systems; these sibling planets also tend to be systematically low in mass - the median $M \sin i=23.5 M_{\oplus}$. In contrast, the Jovian subsample includes six companions, spanning a range from $0.58 M_{\text {Jup }}$ to about $4 M_{\text {Jup }}$ with a median $M \sin i$ of $2.6 M_{\mathrm{Jup}}$.

Is the dramatic difference in the architecture of low-mass planets a bias in Doppler detection efficiency or the result of nature (e.g., conditions in the protoplanetary disk or evolutionary processes)? In Figure 12, we compare the stellar hosts for the super-Earth and Jovian subsamples. The histogram of stellar mass for the hosts of super-Earths is offset to lower mass, with an average of $0.7 M_{\odot}$, while the host stars of the Jovian sample have a mean stellar mass of $1.06 M_{\odot}$. The dependence of reflex velocity on stellar mass implies that the Doppler signals for a star with a mass of $0.7 M_{\odot}$ would be amplified by about $30 \%$ relative to a star of $1.06 M_{\odot}$. Therefore, the paucity of Jupiterlike planets around lower mass stars (Fischer et al. 2011) cannot be a selection effect.

However, assessing the scarcity of super-Earths around the more massive host stars of Jupiter mass planets is complicated by observational detectability issues. The mean velocity amplitude of the super-Earth sample would drop from $4.2 \mathrm{~m} \mathrm{~s}^{-1}$ for the mean host star mass of $0.7 M_{\odot}$ to $3.2 \mathrm{~m} \mathrm{~s}^{-1}$ around solar mass stars. At the same time, as the stellar mass increases from $0.7 M_{\odot}$ to $1.06 M_{\odot}$, the minimum stellar jitter increases by a factor of two (Isaacson \& Fischer 2010; Lovis et al. 2011) or more for chromospherically active stars. As a result, if stars with close-in Jovian-mass planets also host a system of super-Earths, the Doppler signal would be roughly a $1 \sigma$ detection. Furthermore, the observational biases that influence second-planet detection are complex. In some cases the presence of one planet can complicate the detection of additional, lower amplitude planets (for instance if the planets are in resonance (Anglada-Escudé et al. 2010) if one planet has poorly constrained orbital parameters, or if the observational cadence causes aliased signals near the orbital frequency of the additional planet). On the other hand, the presence of a gas giant planet can also cause a star to receive additional observations in preparation for publication, making the detection of low-amplitude planets more likely.

Raymond (2008) finds that although the migration of giant planets does not completely impede terrestrial planet growth, the final accretion phase of terrestrial planets is affected by gravitational perturbations from gas giant planets. Although the Doppler detections may only weakly constrain the presence of low-mass planet siblings to Jupiter mass planets, the Kepler data provide additional support for this case. Latham et al. (2011) proposed that the transiting Jovian planets detected by Kepler would have migrated into their current locations and likely destabilize the orbits of smaller planets. Likewise, they note that many of the Jovian planets detected by Doppler surveys are probably migrated planets that may have destabilized the orbits of close-in Neptunes.

We gratefully acknowledge the dedication and support of the Keck Observatory staff, especially Grant Hill and Scott Dahm for support of HIRES and Greg Wirth for support of remote observing. D.A.F. acknowledges research support from NSF grant AST-1036283 and NASA grant NNX08AF42G. This research has made use of the Exoplanet Orbit Database at exoplanets.org and NASAs ADS Bibliographic Services. Data presented herein were obtained at the W. M. Keck Observatory from telescope time allocated to Yale University and to the National Aeronautics and Space Administration through the agency's scientific partnership with the California Institute of Technology and the University of California and to the University of Hawaii. This research has made use of the SIMBAD database, operated at CDS, Strasbourg, France, and of NASA's Astrophysics Data System Bibliographic Services. The Observatory was made possible by the generous financial support of the W. M. Keck Foundation. The authors wish to recognize and acknowledge the very significant cultural role and reverence that the summit of Mauna Kea has always had within the indigenous Hawaiian community. We are most fortunate to have the opportunity to conduct observations from this mountain.

\section{REFERENCES}

Anglada-Escudé, G., López-Morales, M., \& Chambers, J. E. 2010, ApJ, 709, 168

Apps, K., Clubb, K. I., Fischer, D. A., et al. 2010, PASP, 122, 156

Borucki, W. J., Koch, D. G., Basri, G., et al. 2011, ApJ, 728, 117

Butler, R. P., Johnson, J. A., Marcy, G. W., et al. 2006, PASP, 118, 1685

Butler, R. P., Marcy, G. W., Williams, E., et al. 1996, PASP, 108, 500

Chambers, J. E. 1999, MNRAS, 304, 793

Cumming, A., Butler, R. P., Marcy, G. W., et al. 2008, PASP, 120, 531

Demarque, P., Woo, H.-H., Kim, Y.-C., \& Yi, S. K. 2004, ApJS, 155, 667

Duncan, D. K., Vaughan, A. H., Wilson, O. C., et al. 1991, ApJS, 76, 383

Endl, M., Cochran, W. D., Tull, R. G., \& MacQueen, P. J. 2003, AJ, 126, 3099

ESA 1997, The Hipparcos and Tycho Catalogs (ESA-SP 1200; Noordwijk: ESA Publications Division)

Fischer, D. A., Gaidos, E., \& Brewer, J. 2011, ApJ, submitted

Giguere, M. J., Fischer, D. A., Howard, A. W., et al. 2012, ApJ, in press

Gould, A., Dong, S., Gaudi, B. S., et al. 2010, ApJ, 720, 1073

Gray, R. O., Corbally, C. J., Garrison, R. F., McFadden, M. T., \& Robinson, P. E. 2003, AJ, 126, 2048

Howard, A. W., Marcy, G. W., Bryson, S. T., et al. 2011, ApJ, in press (arXiv:1103.2541)

Isaacson, H., \& Fischer, D. A. 2010, ApJ, 725, 885

Johnson, J. A., Howard, A. W., Marcy, G. W., et al. 2010, PASP, 122, 149

Kasting, J. F., Whitmire, D. P., \& Reynolds, R. T. 1993, Icarus, 101, 108

Latham, D. W., Rowe, J. F., Quinn, S. N., et al. 2011, ApJ, 732, L24

Lepine, S., \& Gaidos, E. 2011, AJ, 142, 138

Lepine, S., \& Shara, M. M. 2005, AJ, 129, 1483

Lovis, C., Dumusque, X., Santos, N. C., et al. 2011, arXiv:1107.5325v1

Marcy, G. W., Butler, R. P., Vogt, S. S., et al. 2005, ApJ, 619, 570

Noyes, R. W., Hartmann, L., Baliunas, S. L., Duncan, D. K., \& Vaughan, A. H. 1984, ApJ, 279, 763

Raymond, S. 2008, in Proc. International Astronomical Union (2007), Vol. 3, 233 
Schlaufman, K. C., \& Laughlin, G. 2011, ApJ, 738, 177

Valenti, J. A., \& Fischer, D. A. 2005, ApJS, 159, 141

Valenti, J. A., Fischer, D. A., Marcy, G. W., et al. 2009, ApJ, 702, 989

Valenti, J. A., \& Piskunov, N. 1996, A\&AS, 118, 595

van Leeuwen, F. 2007, A\&A, 474, 653
Vogt, S. S., Allen, S. L., Bigelow, B. C., et al. 1994, Proc. SPIE, 2198 362

Wright, J. T., Fakhouri, O., Marcy, G. W., et al. 2011, PASP, 123, 412

Wright, J. T., \& Howard, A. 2009, ApJS, 182, 205

Wright, J. T., Upadhyay, S., Marcy, G. W., et al. 2009, ApJ, 693, 1084 